\title{
Aero- $\mathrm{Ga}_{2} \mathrm{O}_{3}$ Nanomaterial Electromagnetically Transparent from Microwaves to Terahertz for Internet of Things Applications
}

\author{
Tudor Braniste ${ }^{1, *(\mathbb{D}}$, Mircea Dragoman ${ }^{2}$, Sergey Zhukov ${ }^{3}$, Martino Aldrigo ${ }^{2}$,

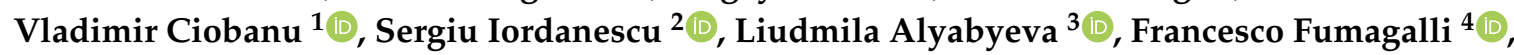 \\ Giacomo Ceccone ${ }^{4}$, Simion Raevschi ${ }^{5}$, Fabian Schütt ${ }^{6}$, Rainer Adelung ${ }^{6}$, Pascal Colpo ${ }^{4}$, \\ Boris Gorshunov ${ }^{3}$ (D) and Ion Tiginyanu $1,7, *$ (D) \\ 1 National Center for Materials Study and Testing, Technical University of Moldova, Stefan cel Mare av. 168, \\ 2004 Chisinau, Moldova; vladimir.ciobanu@cnstm.utm.md \\ 2 National Institute for Research and Development in Microtechnologies (IMT Bucharest), \\ Erou Iancu Nicolae Street 126A, 077190 Voluntari, Romania; mircea.dragoman@imt.ro (M.D.); \\ martino.aldrigo@gmail.com (M.A.); sergiu.iordanescu@imt.ro (S.I.) \\ 3 Laboratory of Terahertz Spectroscopy, Center for Photonics and 2D Materials, Moscow Institute of Physics \\ and Technology (State University), 9 Institutskiy per., 141701 Dolgoprudny, Russia; zs1978@mail.ru (S.Z.); \\ aliabeva.ln@mipt.ru (L.A.); gorshunov.bp@mipt.ru (B.G.) \\ 4 European Commission, Joint Research Centre (JRC), Via E. Fermi, 2749, 21027 Ispra, Italy; \\ francesco-sirio.fumagalli@ec.europa.eu (F.F.); giacomo.ceccone@ec.europa.eu (G.C.); \\ pascal.colpo@ec.europa.eu (P.C.) \\ 5 Department of Physics and Engineering, State University of Moldova, Alexei Mateevici str. 60, \\ 2009 Chisinau, Moldova; raevskis@mail.ru \\ 6 Institute for Materials Science, Kiel University, Kaiserstr. 2, D-24143 Kiel, Germany; fas@tf.uni-kiel.de (F.S.); \\ ra@tf.uni-kiel.de (R.A.) \\ 7 Academy of Sciences of Moldova, Stefan cel Mare av. 1, MD-2001 Chisinau, Moldova \\ * Correspondence: tudor.braniste@cnstm.utm.md (T.B.); tiginyanu@asm.md (I.T.); Tel.: +373-22-509-920 (I.T.)
}

Received: 4 May 2020; Accepted: 27 May 2020; Published: 29 May 2020

\begin{abstract}
In this paper, fabrication of a new material is reported, the so-called Aero- $\mathrm{Ga}_{2} \mathrm{O}_{3}$ or Aerogallox, which represents an ultra-porous and ultra-lightweight three-dimensional architecture made from interconnected microtubes of gallium oxide with nanometer thin walls. The material is fabricated using epitaxial growth of an ultrathin layer of gallium nitride on zinc oxide microtetrapods followed by decomposition of sacrificial $\mathrm{ZnO}$ and oxidation of $\mathrm{GaN}$ which according to the results of $\mathrm{X}$-ray diffraction (XRD) and X-ray photoemission spectroscopy (XPS) characterizations, is transformed gradually in $\beta-\mathrm{Ga}_{2} \mathrm{O}_{3}$ with almost stoichiometric composition. The investigations show that the developed ultra-porous Aerogallox exhibits extremely low reflectivity and high transmissivity in an ultrabroadband electromagnetic spectrum ranging from X-band $(8-12 \mathrm{GHz})$ to several terahertz which opens possibilities for quite new applications of gallium oxide, previously not anticipated.
\end{abstract}

Keywords: aero- $\mathrm{Ga}_{2} \mathrm{O}_{3}$; ultra-porous nanomaterial; extremely low reflectivity; electromagnetically transparent nanomaterial; $\mathrm{X}$-band and terahertz frequencies

\section{Introduction}

The materials transparent for a certain electromagnetic bandwidth are key components for many industries such as aeronautic, space, telecommunications, etc. [1,2]. They are called radomes and are configured in the form of various enclosures depending on the applications; their role is to protect antennas from various agents such as rain, snow, dust, heat, etc. The radomes can be seen, for example, 
in any airport or on top of high buildings. The new wireless communication systems imply that very high frequencies will be used such as $0.1 \mathrm{THz}$ for $5 \mathrm{G}$ and $10 \mathrm{THz}$ for $6 \mathrm{G}$ [3]. There are materials which are transparent in the $\mathrm{THz}$ region, such as high resistivity (HR) semiconductors (silicon, boron nitride, gallium arsenide, germanium) or dielectrics (quartz, sapphire, fused silica, diamonds). However, for Internet of Things (IoTs) applications [4], which are the backbone of 5G and 6G communications, very small and lightweight enclosures are required to protect antennas, since the dimensions of antennas are reduced to tens of microns and even lower, comparable to the dimensions of a human hair. Thus, the existing materials for radomes at airports, telecommunications and in many other applications could not be directly reused for tiny IoT due to the requirements of dimensions and weight and there is a real need to find new transparent materials for the applications involved.

The wide bandgap $\beta-\mathrm{Ga}_{2} \mathrm{O}_{3}$ semiconductor is studied intensively for power electronics [5]. Please note that along with $\beta-\mathrm{Ga}_{2} \mathrm{O}_{3}$, there are other $\mathrm{Ga}_{2} \mathrm{O}_{3}$ polymorphs, all with a small index of refraction (less than 2) [6], indicating that gallium oxide could be a promising candidate for use in transparent electronics. However, little is known about RF properties of $\mathrm{Ga}_{2} \mathrm{O}_{3}$ since only a few results on $\mathrm{Ga}_{2} \mathrm{O}_{3}$ transistors are reported (see [7] and the references therein). Taking into account that three-dimensional architectures consisting of networks of low-dimensional structures prove to be among the multifunctional materials most promising for new applications in electronics and biomedicine [8,9], we developed an ultra-porous architecture made from interconnected microtubes of $\beta-\mathrm{Ga}_{2} \mathrm{O}_{3}$ with nanometer thin walls, carried out its structural characterization and experimentally demonstrated that the new ultra-lightweight nanomaterial, called aero- $\mathrm{Ga}_{2} \mathrm{O}_{3}$ or Aerogallox, is highly transparent and exhibits extremely low reflectivity in the X-band and THz region, up to $3 \mathrm{THz}$, thus disclosing a novel application of $\mathrm{Ga}_{2} \mathrm{O}_{3}$ previously not anticipated.

\section{Materials and Methods}

The technological route for the fabrication of aero- $\mathrm{Ga}_{2} \mathrm{O}_{3}$ is as follows. Initially the aero-GaN was obtained by growing an ultra-thin layer of $\mathrm{GaN}$ on sacrificial $\mathrm{ZnO}$ templates [10]. The $\mathrm{ZnO}$ templates represented networks of interpenetrated $\mathrm{ZnO}$ microtetrapods obtained using the flame transport synthesis approach, as previously described in ref. [11]. GaN was grown in a hydride vapor phase epitaxy (HVPE) horizontal reactor containing distinct source and reaction zones. Metallic gallium as well as ammonia $\left(\mathrm{NH}_{3}, 99.99 \%\right.$, EG No. 231-653.3), hydrogen chloride ( $\left.\mathrm{HCl}, 99.9995 \%\right)$ and hydrogen $\left(\mathrm{H}_{2}, 99.999 \%\right)$ acquired from Geschaftsbereich Linde Gas, Germany, were used as source materials and carrier gases during the growth process. In the source zone, at high temperature $\left(T=850{ }^{\circ} \mathrm{C}\right)$ the $\mathrm{GaCl}$ is formed as a result of chemical reactions between gaseous $\mathrm{HCl}$ and liquid $\mathrm{Ga}$. The gaseous $\mathrm{GaCl}$ and $\mathrm{NH}_{3}$ reacted with each other in the react zone, where initially the temperature was kept at $600{ }^{\circ} \mathrm{C}$ for $10 \mathrm{~min}$ to initiate nucleation of $\mathrm{GaN}$ on the surface of $\mathrm{ZnO}$ microtetrapods, and then increased up to $T_{\mathrm{g}}=850{ }^{\circ} \mathrm{C}$ for $10 \mathrm{~min}$ to produce GaN layer. In the process of $\mathrm{GaN}$ growth, the $\mathrm{HCl}, \mathrm{NH}_{3}$ and $\mathrm{H}_{2}$ flow rates were equal to 15,600 and $3600 \mathrm{smL} / \mathrm{min}$, respectively. In the process of HVPE growth of GaN, the $\mathrm{ZnO}$ sacrificial template is being decomposed due to corrosive atmosphere at high temperature leading to the formation of microtubular structures representing aero-GaN [10]. Previously, we demonstrated the high crystalline quality of the resulting $\mathrm{GaN}$ microtubes as well as the existence of $\mathrm{ZnO}$ traces (at the level of about $2 \%$ ) on the inner surface of GaN microtube walls [10,12]. At the final step of the technological route, the aero-GaN is subjected to annealing in air at $950{ }^{\circ} \mathrm{C}$ for $60 \mathrm{~min}$ and, as a result, is transformed into aero- $\mathrm{Ga}_{2} \mathrm{O}_{3}$ or Aerogallox.

The aero-GaN and aero- $\mathrm{Ga}_{2} \mathrm{O}_{3}$ thin films crystal structure and phases were investigated using a Bruker AXS D8 Advance X-ray diffractometer (XRD, Bruker Italia S.r.1., Milano, Italy) in a standard $\theta-2 \theta$ Bragg-Brentano configuration with a monochromatic $\mathrm{Cu} \mathrm{K} \alpha 1(\lambda=0.15406 \AA)$ radiation. A $40 \mathrm{kV}$ beam voltage and $40 \mathrm{~mA}$ beam current were used. For acquisition a linear position-sensitive semiconductor detector (LYNXEYE, Bruker Italia S.r.1., Milan, Italia) in 0D-mode was used, beam optics were Göbel mirror, $6 \mathrm{~mm}$ slit, Soller $2.5^{\circ}$; detector optics were $6 \mathrm{~mm}$ slit, Soller $2.5^{\circ}$. Diffraction 
pattern data were collected between $20^{\circ}$ and $50^{\circ}$ with step lengths of $0.025^{\circ}$, the sample was measured in powder form.

The chemical composition at surfaces was studied by means of X-ray Photoemission Spectroscopy, XPS (AXIS ULTRA, DLD Kratos Analytical, Manchester, UK) equipped with a monochromatic Al $\mathrm{K} \alpha$ source $(\mathrm{h} v=1486.6 \mathrm{eV})$ operating at $150 \mathrm{~W}$ (h-Planck's constant, $v$-frequency). Spectra were recorded from a $100 \times 100 \mu \mathrm{m}^{2}$ analysis area and at $160 \mathrm{eV}$ (survey) pass energy, whereas core level spectra were recorded using pass energy of $20 \mathrm{eV}$. Operating pressure was $6 \times 10^{-7} \mathrm{~Pa}$. Prior to the measurement, the surfaces were sputtered using an $\mathrm{Ar}^{+}$beam operated at $2 \mathrm{keV}$ and $0.84 \mu \mathrm{A}$ for $2 \mathrm{~min}$. Surface charging was compensated using low energy $(\sim 5 \mathrm{eV})$ electrons and adjusted using the charge balance plate on the instrument. Three different spots were analyzed for each sample. All the spectra were processed with CasaXPS (ver. 2.3.20). Spectra were calibrated setting hydrocarbon C1s at $285.0 \mathrm{eV}$. The surface composition was evaluated from the survey spectra, after a Tougaard U3-type background subtraction, using relative sensitivity factors provided by the manufacturer. Peak fitting was performed with no preliminary smoothing. Symmetric Gaussian-Lorentzian (70\% Gaussian and $30 \%$ Lorentzian) product functions were used to approximate the line shapes of the fitting components.

For the microwaves and terahertz characterizations of the material, bulk samples were prepared in the form of rectangular pellets $(20 \mathrm{~mm} \times 10 \mathrm{~mm} \times 2 \mathrm{~mm}$ ). The freestanding samples were exposed to electromagnetic radiation. The microwave characterization of the aero- $\mathrm{Ga}_{2} \mathrm{O}_{3}$ pellets was carried out by means of a VNA (Vector Network Analyzer) connected to a WR90 waveguide-based set-up suitable for measurements in the X-band (i.e., 8.2-12.4 GHz) [13]. A schematics of the waveguide is presented in Figure 1, where $a=22.86 \mathrm{~mm}$ and $b=10.16 \mathrm{~mm}$. Since the dimensions of the cavity are a bit larger than the aero- $\mathrm{Ga}_{2} \mathrm{O}_{3}$ pellet, we made use of a supporting flange to fix the sample in the cavity, as to avoid electromagnetic radiation due to imperfect coupling between the two waveguides, hence affecting the reliability of the performed measurements.

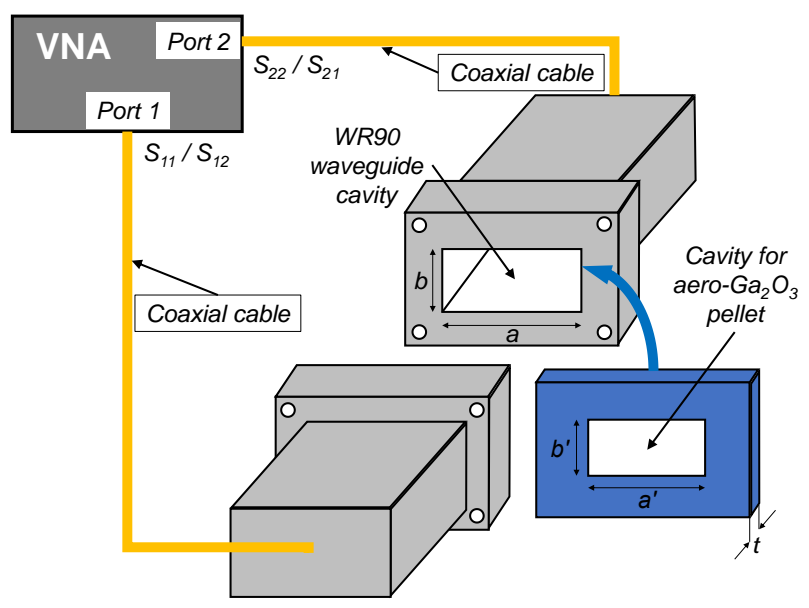

Figure 1. Microwave experimental set-up for X-band characterization of the aero- $\mathrm{Ga}_{2} \mathrm{O}_{3}$.

For terahertz measurements the samples were fixed on metallic holders covering $6 \mathrm{~mm}$ aperture, the radiation passing through the sample in free space. Samples with different densities from 70 to 110 $\mathrm{mg} / \mathrm{cm}^{3}$ of aero- $\mathrm{Ga}_{2} \mathrm{O}_{3}$ were investigated. Room-temperature terahertz spectra of complex dielectric permittivity $\varepsilon^{*}(v)=\varepsilon^{\prime}(v)+\mathrm{i} \varepsilon^{\prime \prime}(v)$ were measured in the range $v=10-100 \mathrm{~cm}^{-1}$ in a quasi-optical arrangement (in an open space with no waveguides used) with the help of commercial time-domain spectrometer TeraView TPS 3000 (TeraView, Cambridge, UK). The spectra of the real and imaginary parts of dielectric permittivity are determined in the transmission geometry via measurements of the complex transmission coefficient (amplitude and phase) of the plane-parallel samples; standard expressions for electrodynamics of a plane-parallel layer are used [14]. In addition, the spectra of 
transmission coefficients of the same samples were measured at frequencies up to $7000 \mathrm{~cm}^{-1} \mathrm{using}^{\mathrm{a}}$ standard Fourier-transform spectrometer Bruker Vertex 80v.

\section{Results and Discussion}

\subsection{Structural Characterization of Aero $-\mathrm{Ga}_{2} \mathrm{O}_{3}$}

Figure 2 depicts scanning electron microscope (SEM) images of interpenetrated networks of $\mathrm{ZnO}$ microtetrapods (a), aero-GaN (b) and the obtained aero- $\mathrm{Ga}_{2} \mathrm{O}_{3}$ (c). The inset pictures are the respective photos of the pellets of $20 \mathrm{~mm} \times 10 \mathrm{~mm} \times 2 \mathrm{~mm}(\mathrm{~L} \times \mathrm{W} \times \mathrm{H})$, where one can easily distinguish the change in color of the material after each technological step (white, yellow, white).
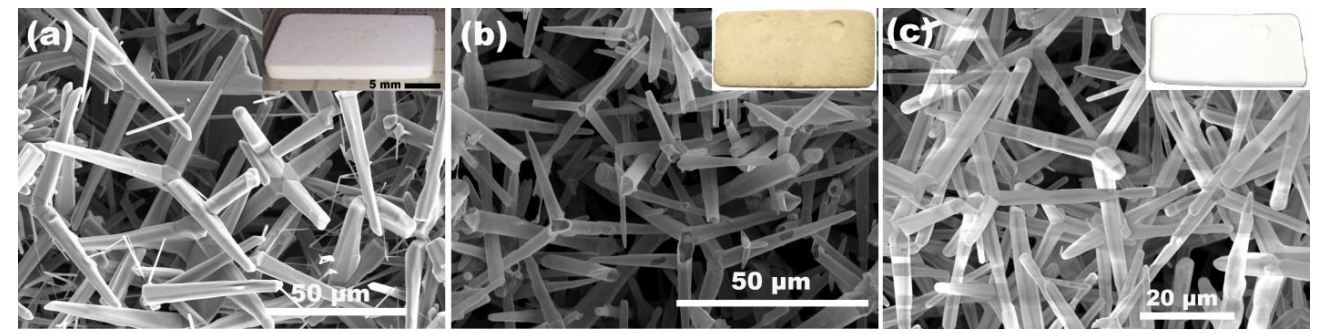

Figure 2. SEM images of (a) initial $\mathrm{ZnO}$ template, (b) intermediate aero-GaN, and (c) resulted aero- $\mathrm{Ga}_{2} \mathrm{O}_{3}$ nanomaterial. The inset pictures represent the photographs of the pellet samples of $\mathrm{ZnO}$, $\mathrm{GaN}$, and $\mathrm{Ga}_{2} \mathrm{O}_{3}$ respectively.

Figure 3 illustrates the XRD spectra of aero-GaN (Figure 3a) used for the preparation of aero- $\mathrm{Ga}_{2} \mathrm{O}_{3}$, the mixture phase of $\mathrm{GaN}$ and $\mathrm{Ga}_{2} \mathrm{O}_{3}$ (Figure $3 \mathrm{~b}$ ) that was obtained by thermal treatment of aero-GaN at $T=800{ }^{\circ} \mathrm{C}$ in air for $1 \mathrm{~h}$, and the completely transformed aero- $\mathrm{Ga}_{2} \mathrm{O}_{3}$ (Figure $3 \mathrm{c}$ ) obtained after $1 \mathrm{~h}$ of treatment at $950^{\circ} \mathrm{C}$ of aero-GaN samples. The XRD reflections at $32.25^{\circ}, 36.8^{\circ}$ and $48.12^{\circ}$ were assigned to wurtzite GaN planes (010), (011) and (012), respectively, while the reflections at $30.3^{\circ}, 31.7^{\circ}, 33.5^{\circ}$, $35.3^{\circ}, 38.4^{\circ}, 43.2^{\circ}, 45.9^{\circ}$ and $48.9^{\circ}$ were assigned to beta phase of monoclinic $\mathrm{Ga}_{2} \mathrm{O}_{3}$ planes $(-110),(002)$, $(-1-11),(1-11),(202),(600),(1-12)$ and (-510), respectively. Comparing the different patterns, the qualitative trend of GaN oxidative phase change occurring at different stages of the thermal synthesis processes can be appreciated. While both nitride and oxide phases co-exist after the thermal step at $T=800^{\circ} \mathrm{C}$ in air for $1 \mathrm{~h}$ (both reflection peaks sets for wurtzite $\mathrm{GaN}$ and monoclinic $\mathrm{Ga}_{2} \mathrm{O}_{3}$ are found in the diffraction pattern), only reflections from the oxide remain after annealing at $T=950^{\circ} \mathrm{C}$. Formation of monoclinic $\mathrm{Ga}_{2} \mathrm{O}_{3}$ in thin structures was already observed for annealing temperatures as low as $750{ }^{\circ} \mathrm{C}[15]$.

Figure 4 shows the principal results of $\beta-\mathrm{Ga}_{2} \mathrm{O}_{3}$ surface chemical analysis by means of XPS. Survey spectra (Figure 4a) show that the main elements left in the sample after annealing are $\mathrm{Ga}$ and $\mathrm{O}$ with low level of $C$ and $\mathrm{Zn}$ present as impurities. Observed gallium main emission lines are Ga $2 p$ (shown in Figure 4b), Ga 3p and Ga 3d (not shown) and several Auger lines in the 400-600 eV regions. All Ga doublets show energy shifts with respect to metallic gallium binding energy (BE), consistent with literature reported values for $\beta-\mathrm{Ga}_{2} \mathrm{O}_{3}[16,17]$. Data show that $\mathrm{O} 1 \mathrm{~s}$ peak (Figure $4 \mathrm{c}$ ) need to be resolved using two components. The main component $\mathrm{BE}$ at $530.98 \mathrm{eV}$ can be attributed to $\mathrm{Ga}-\mathrm{O}$ bonding in the oxide while the higher $\mathrm{BE}$ contribution at $533.17 \mathrm{eV}$ can be assigned to $\mathrm{O}$-vacancies sites and/or Ga suboxides [18-20]. This peak presence is related to the Ar ions sputtering process used to clean the sample surface in order to remove adventitious carbon contamination. Presence of reduced Ga can be seen also in the low energy satellite peak (at BE $19.16 \mathrm{eV}$ ) of Ga 3d (not shown). However, sputter cleaning of the sample was necessary for reliable estimation of atomic concentrations; before $\mathrm{Ar}^{+}$bombardment $\mathrm{C}$ atoms surface concentration was estimated to be around $11 \%$ while after cleaning dropped down to $2 \%$. Measured Ga/O ratio is thus 0.62 with an estimated relative error of $6.21 \%$ (stoichiometric value 0.67$)$. The presence of $\mathrm{Zn} 2 \mathrm{p}$ doublet (Figure $4 \mathrm{~d}$ ) suggests some residual $(<1$ at. \%) of metallic impurities left from the sacrificial $\mathrm{ZnO}$ matrix used in the fabrication process. It is to 
be noted that the chemical composition study using energy dispersive $\mathrm{X}$-ray analysis disclosed the stoichiometric composition of the $\mathrm{Ga}_{2} \mathrm{O}_{3}$ nanoarchitecture, at the same time traces of Zinc at the level of about 1.5 at. \% were shown.

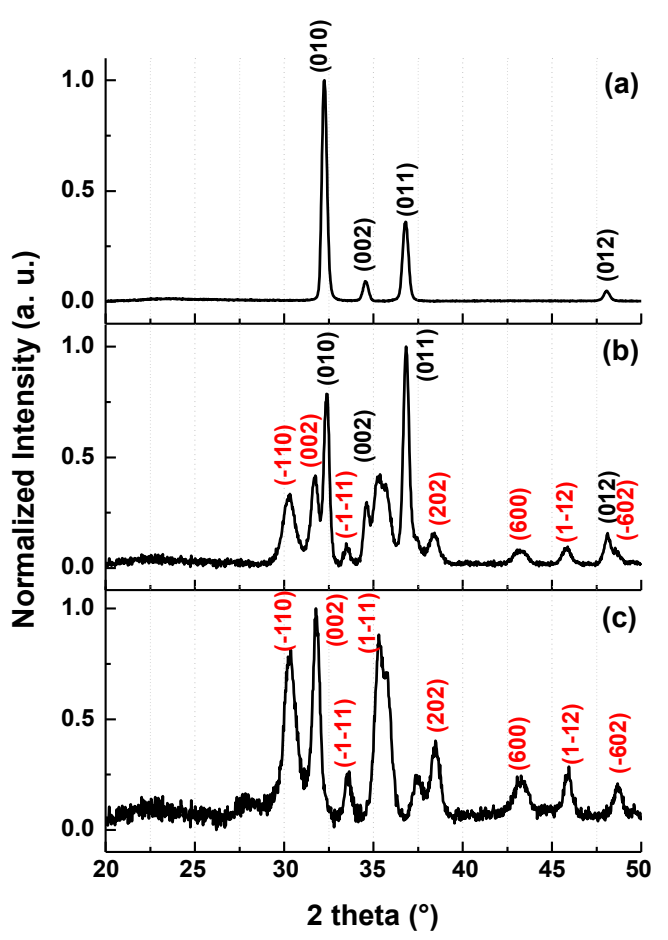

Figure 3. XRD spectra of aero-GaN (a), mixture of $\mathrm{GaN}$ and $\mathrm{Ga}_{2} \mathrm{O}_{3}$ phases (b) and aero- $\mathrm{Ga}_{2} \mathrm{O}_{3}$ (c).

(a)
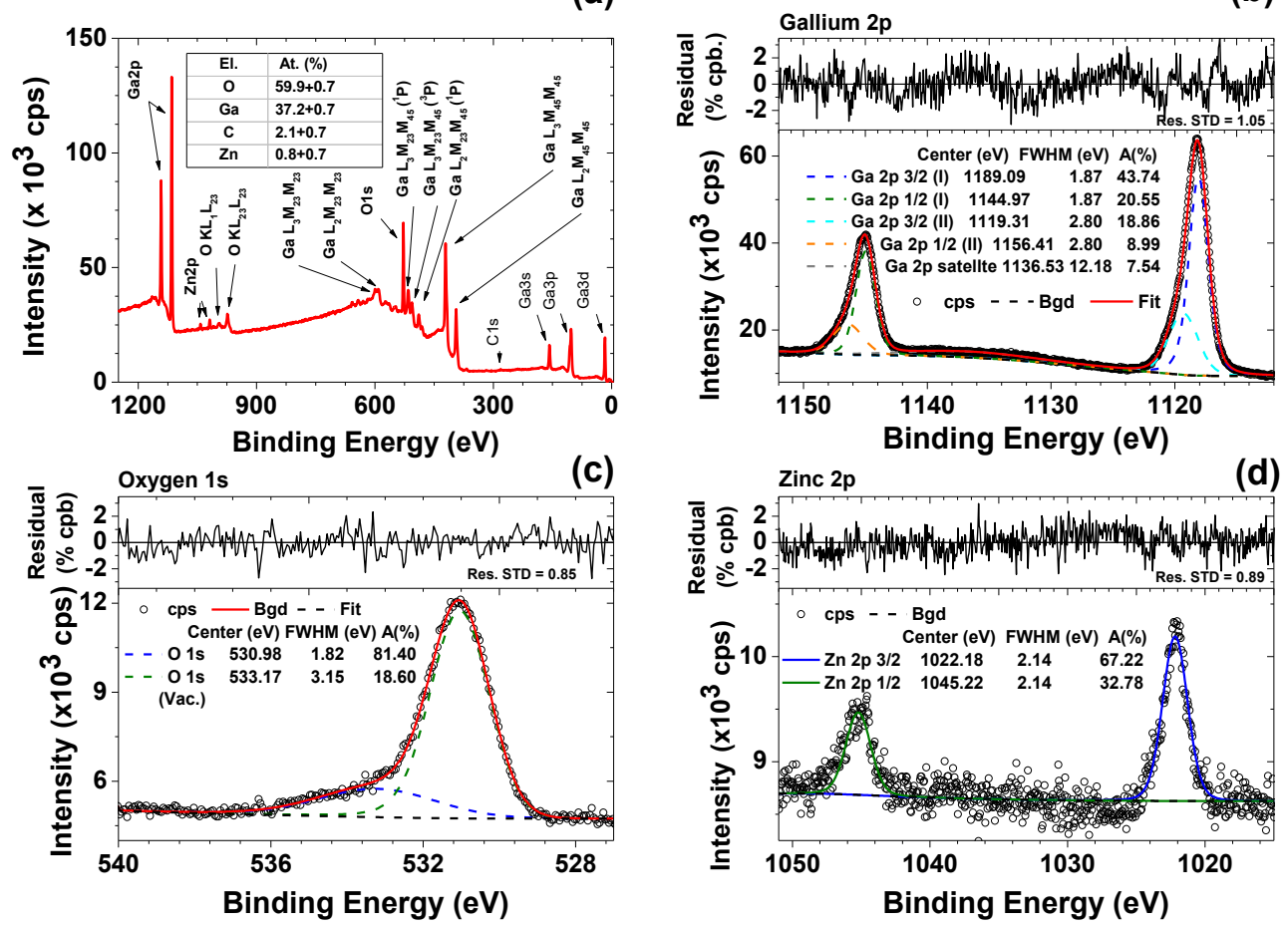

Figure 4. (a) XPS survey spectra of $\beta-\mathrm{Ga}_{2} \mathrm{O}_{3}$ powder with attribution of the principal emission lines, table in the inset shows the derived atomic abundancies. (b-d) High resolution XSP spectra with resolved peak components and normalized residuals, of the Ga 2p (b), O 1s (c) and Zn 2p (d) regions. 


\subsection{Characterization of Aero- $\mathrm{Ga}_{2} \mathrm{O}_{3}$ at Microwaves.}

The S-parameters at the two ports of the VNA were measured to provide the reflection $\left(\mathrm{S}_{11} / \mathrm{S}_{22}\right)$ and transmission $\left(\mathrm{S}_{12} / \mathrm{S}_{21}\right)$ coefficients at/between the two ports, respectively. The measured S-parameters of the Aerogallox sample (Figure 2c) with the density of $110 \mathrm{mg} / \mathrm{cm}^{3}$ are depicted in Figure 5.
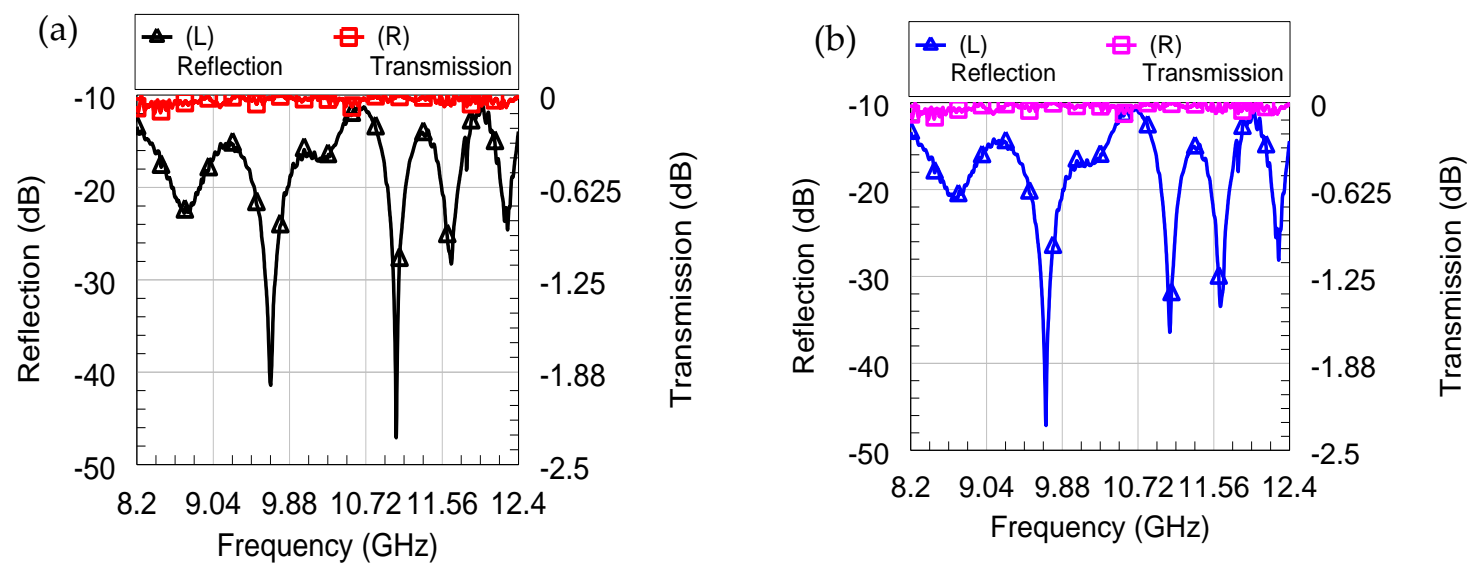

Figure 5. Microwave measurements in X-band for the two cases: (a) without the aero- $\mathrm{Ga}_{2} \mathrm{O}_{3}$ pellet and (b) with the aero- $\mathrm{Ga}_{2} \mathrm{O}_{3}$ pellet, in terms of reflection (left vertical axis, solid black and blue curves) and transmission (right vertical axis, solid red and pink curves).

In Figure 5a,b we show only $S_{11}$ and $S_{21}$, since $S_{22}$ is identical with $S_{11}$, and $S_{12}$ with $S_{21}$ thanks to reciprocity and symmetry of the scattering matrix (typical for passive components). One can notice that the reflection coefficient is better than $-10 \mathrm{~dB}$ all over the band of interest, whereas the insertion loss has a maximum value better than $-0.12 \mathrm{~dB}$ in both cases without and with aero- $\mathrm{Ga}_{2} \mathrm{O}_{3}$. In other words, the presence of the aero- $\mathrm{Ga}_{2} \mathrm{O}_{3}$ inside the cavity between the two X-band waveguides does not affect at all the transmission of the microwave signal, which means the aero- $\mathrm{Ga}_{2} \mathrm{O}_{3}$ is completely (within our accuracy) electromagnetically transparent in the X-band.

\subsection{Characterization of Aero- $\mathrm{Ga}_{2} \mathrm{O}_{3}$ in the Terahertz Region}

Two samples of Aerogallox with different densities were studied-the low density equals to 70 $\mathrm{mg} / \mathrm{cm}^{3}$ and the high density was $110 \mathrm{mg} / \mathrm{cm}^{3}$. Comparative analyses with aero-GaN samples with the density of $15 \mathrm{mg} / \mathrm{cm}^{3}$ were performed. Broad-band terahertz-infrared transmissivity spectra for two samples with low and high densities are shown in Figure 6a. As expected, more dense samples have lower transmissivity. A decrease in the transmission coefficient for frequencies growing up to $200 \mathrm{~cm}^{-1}$ is caused by intensive absorption between 200 and $800 \mathrm{~cm}^{-1}$ (see inset in Figure 6a). Our attempts to extract information about absorption mechanisms in this range by measuring the spectra of reflection coefficient failed due to very low intensity reflected by the samples, i.e., extremely small value of reflectivity, as discussed below. Below $200 \mathrm{~cm}^{-1}$, there are two narrow absorption resonances located at $\sim 155$ and $\sim 176 \mathrm{~cm}^{-1}$ whose parameters (frequency position and intensity) do not depend noticeably on the sample density. A few more resonances are observed above $800 \mathrm{~cm}^{-1}$, most intensive at $\sim 3500 \mathrm{~cm}^{-1}$. Origin of observed narrow absorptions seems to be related to the presence of impurities such as hydrogen [21]. 


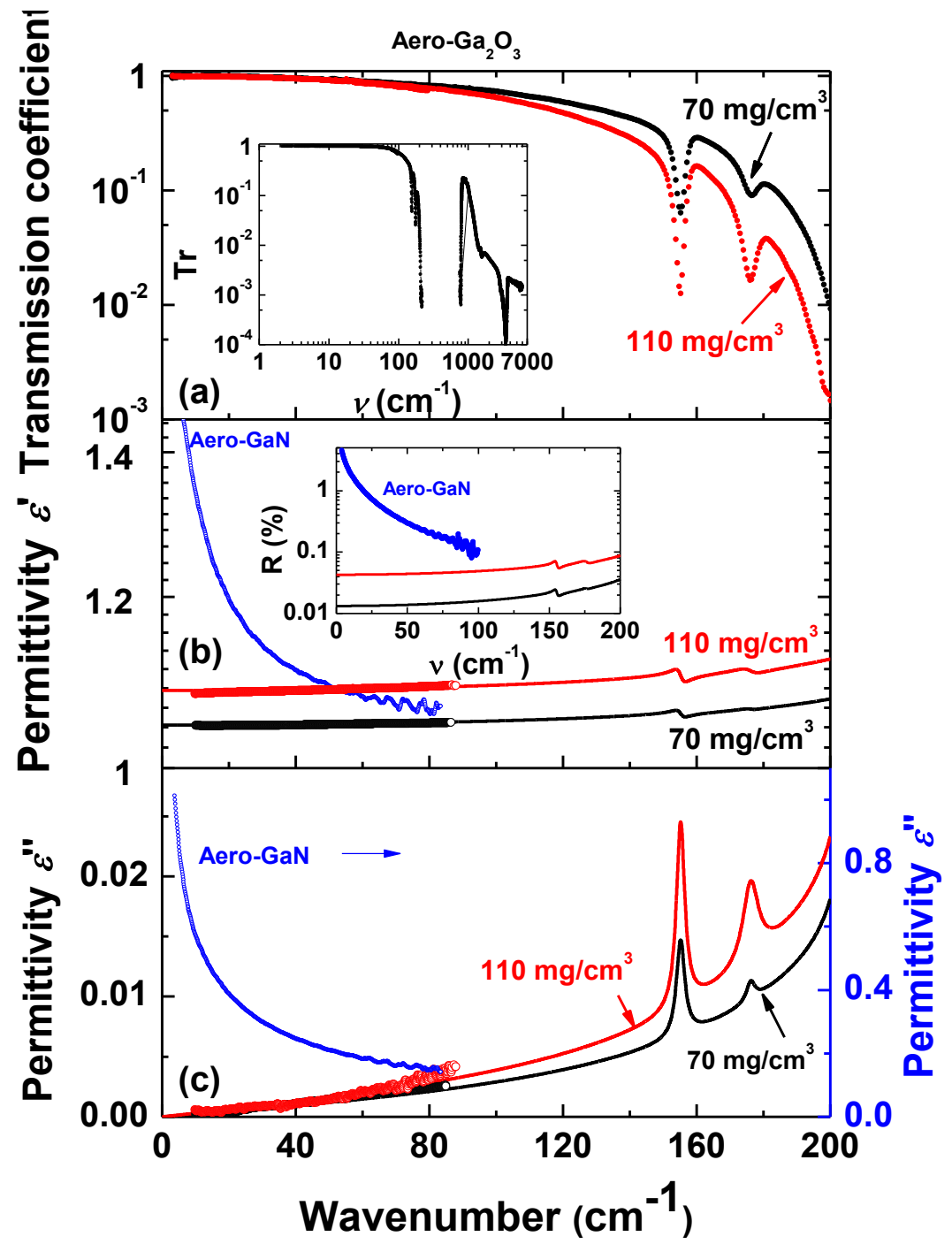

Figure 6. Terahertz spectral characteristics of aero- $\mathrm{Ga}_{2} \mathrm{O}_{3}$ samples with two different densities of $70 \mathrm{mg} / \mathrm{cm}^{3}$ and $110 \mathrm{mg} / \mathrm{cm}^{3}$, and aero-GaN sample with the density of $15 \mathrm{mg} / \mathrm{cm}^{3}$ (data from [22]): transmission coefficient $\operatorname{Tr}(\mathbf{a})$, real $\varepsilon^{\prime}(\mathbf{b})$ and imaginary $\varepsilon^{\prime \prime}(\mathbf{c})$ parts of dielectric permittivity. Open dots on panels (b) and (c) present $\mathrm{THz}$ data for permittivity. Inset in panel (a): spectrum of transmission coefficient measured at frequencies up to $7000 \mathrm{~cm}^{-1}$. Inset in panel (b): spectra of reflection coefficient calculated basing on measured spectra of real and imaginary parts of dielectric permittivity using standard Fresnel expressions [14].

In Figure $6 \mathrm{~b}, \mathrm{c}$ we present the spectra of real and imaginary permittivity of the two samples, together with the reflection coefficient. It is seen that $\mathrm{THz}$ characteristics of our aero- $\mathrm{Ga}_{2} \mathrm{O}_{3}$ material drastically differ from those of parent bulk $\mathrm{Ga}_{2} \mathrm{O}_{3}$ crystal as well as from any other bulk semiconductor. For example, typical semiconductors $\mathrm{Ge}, \mathrm{GaAs}$ and $\mathrm{Si}$ have refractive indexes at $300 \mathrm{GHz} n=3.99$, 3.59 and 3.43, respectively [23], while the refractive index of aero- $\mathrm{Ga}_{2} \mathrm{O}_{3}, n \approx 1.07$ (sample with density $70 \mathrm{mg} / \mathrm{cm}^{3}$, Figure 7$)$, only slightly exceeds that of vacuum $(n=1)$. From Figure $6 \mathrm{~b}, \mathrm{c}$ one can see that there is pronounced difference between terahertz response of aero- $\mathrm{Ga}_{2} \mathrm{O}_{3}$ and previously studied aero-GaN [22]. While in aero- $\mathrm{Ga}_{2} \mathrm{O}_{3}$ real permittivity $\varepsilon^{\prime}$ is dispersionless and imaginary permittivity $\varepsilon^{\prime \prime}$ is small and approaches zero with frequency decrease, both indicating absence of any absorption process, aero-GaN demonstrates strong increase of $\varepsilon^{\prime}$ and $\varepsilon^{\prime \prime}$ toward low frequencies. The origin of corresponding pronounced absorption is associated with the polarizability of the 3D architecture of mutually interpenetrated $\mathrm{GaN}$ aerotetrapods, with the $\mathrm{ZnO}-\mathrm{GaN}$ interfaces, and finally 
with dynamics of relatively big complexes of tetrapods. No such contribution exists in the present Aerogallox nanomaterial. Aero- $\mathrm{Ga}_{2} \mathrm{O}_{3}$ is characterized by very small imaginary permittivity $\varepsilon^{\prime \prime}$ and dielectric losses $\tan \delta=\varepsilon^{\prime \prime} / \varepsilon^{\prime}$ (Figure 7a) that are responsible for radiation absorption, and by real part of permittivity $\varepsilon^{\prime}$ and real part $n$ of complex refractive index $n^{*}=n+\mathrm{i} k$ (Figure $7 \mathrm{~b}$ ) close to 1 . Both factors lead to a very low reflection coefficient (inset in Figure 6b) $R=\left[(n-1)^{2}+k^{2}\right] /\left[(n+1)^{2}+k^{2}\right] \approx$ $\left[\left(\sqrt{\varepsilon^{\prime}}-1\right)^{2}+k^{2}\right] /\left[(\sqrt{\varepsilon}+1)^{2}+k^{2}\right]$ that is as small as $R \approx 0.1 \%$ and can be made close to $R \approx 0.01 \%$. We can compare these extremely low reflectivity values with those of typical bulk semiconductors, mentioned above, $\mathrm{Ge}(n=3.99)$, GaAs $(n=3.59)$ or silicon $(n=3.43)$; corresponding reflection coefficients fall in the range $30-36 \%$. Along with high value of transmissivity, this low reflectivity of the material may be beneficial for future applications of $\mathrm{Ga}_{2} \mathrm{O}_{3}$.

\section{Frequency $(\mathrm{THz})$}
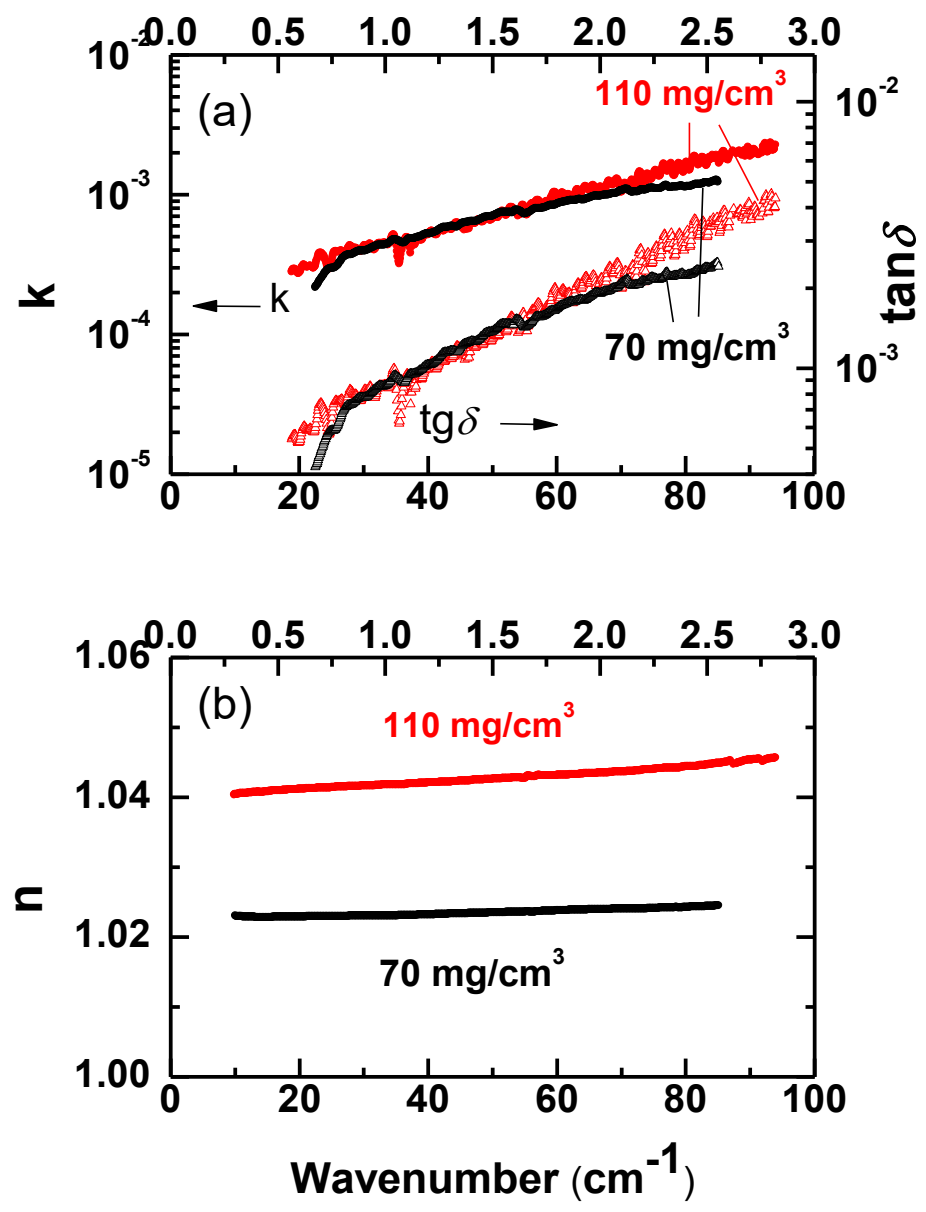

Figure 7. Spectra of terahertz electrodynamic characteristics of aero- $\mathrm{Ga}_{2} \mathrm{O}_{3}$ samples with two different densities of $70 \mathrm{mg} / \mathrm{cm}^{3}$ and $110 \mathrm{mg} / \mathrm{cm}^{3}$ : imaginary $k(\mathbf{a})$ and real $n(\mathbf{b})$ parts of complex refractive index $n+\mathrm{i} k$ and dielectric loss tangent tan $\delta$ (panel a, triangles).

\section{Conclusions}

We developed a highly porous ultra-lightweight three-dimensional nanoarchitecture consisting of interconnected microtubes of $\mathrm{Ga}_{2} \mathrm{O}_{3}$ with nanometer thick walls, and demonstrated that the gallium oxide skeleton is of crystalline $\beta$-phase with almost stoichiometric composition. The new nanomaterial is shown to exhibit ultra-low reflectivity and high transparency in an extremely wide range of the electromagnetic spectrum, covering the X-band and THz region, up to $3 \mathrm{THz}$. The disclosed novel properties of aero- $\mathrm{Ga}_{2} \mathrm{O}_{3}$ open possibilities, in premiere, for the use of gallium oxide in IoT applications. 
Author Contributions: Conceptualization: I.T., M.D., B.G., R.A.; Data curation, T.B., S.Z., M.A., S.I., L.A., V.C., F.F., G.C.; Formal analysis, T.B., M.D., S.Z., B.G., M.A., F.F., G.C.; Funding acquisition, I.T., B.G., P.C., R.A; Investigation, T.B., S.Z., M.A., L.A., S.I., V.C., F.F., G.C., S.R.; Methodology, T.B., M.D., S.Z., B.G., I.T.; Project administration, I.T.; Resources, T.B., F.S., S.R.; Supervision, M.D., B.G, I.T.; Validation, T.B., S.Z., L.A., M.A., S.I., V.C., F.F., G.C., P.C., S.R.; Writing-original draft, M.D., T.B., B.G., I.T.; All authors have read and agreed to the published version of the manuscript.

Funding: The authors acknowledge the support from the Ministry of Education, Culture and Research of the Republic of Moldova under the Grant \#20.80009.50007.20, and to the European Union's Horizon-2020 Research and Innovation Programme: H2020-EU.4.b-Twinning of research institutions, grant agreement No. 810652 "NanoMedTwin". F.S. and R.A. acknowledge funding by the Deutsche Forschungsgemeinschaft (DFG) under contracts AD 183/27-1.

Conflicts of Interest: The authors declare no conflict of interest.

\section{References}

1. Mani, G.S. Radome Materials. In Microwave Materials; Murthy, V.R.K., Sundaram, S., Viswanathan, B., Eds.; Springer: Berlin, Germany, 1994; pp. 200-239.

2. Khatavkara, N.; Balasubramanian, K. Composite materials for supersonic aircraft radomes with ameliorated radio frequency transmission-A review. RCS Adv. 2016, 6, 6709-6718. [CrossRef]

3. Dang, S.; Amin, O.; Shihada, B.; Alouini, M.-S. What should 6G be? Nat. Electron. 2020, 3, 20-29. [CrossRef]

4. Asghari, P.; Rahmani, A.M.; Javadi, H.H.S. Internet of Things applications: A systematic review. Comput. Networks 2019, 148, 241-261. [CrossRef]

5. Zhou, H.; Zhang, J.; Zhang, C.; Feng, Q.; Zhao, S.; Ma, P.; Hao, Y. A review of the most recent progresses of state-of-art gallium oxide power devices. J. Semicond. 2019, 40, 011803. [CrossRef]

6. Pearton, S.J.; Yang, J.; Cary IV, P.H.; Ren, F.; Kim, J.; Tadjer, M.J.; Mastro, M.A. A review of $\mathrm{Ga}_{2} \mathrm{O}_{3}$ materials, processing, and devices. Appl. Phys. Rev. 2018, 5, 011301. [CrossRef]

7. Xia, Z.; Xue, H.; Joishi, C.; McGlone, J.; Kalaricka, N.K.; Sohel, S.H.; Brenner, M.; Arehart, A.; Ringel, S.; Lodha, S.; et al. $\beta-\mathrm{Ga}_{2} \mathrm{O}_{3}$ delta-doped field-effecttransistors with current gain cutoff frequency of $27 \mathrm{GHz}$. IEEE Trans. El. Dev. 2019, 40, 1053-1055.

8. Jakus, A.E.; Secor, E.B.; Rutz, A.L.; Jordan, S.W.; Hersam, M.C.; Shah, R.N. Three-dimensional printing of high-content graphene scaffolds for electronics and biomedical applications. ACS Nano 2015, 9, 4636-4648. [CrossRef] [PubMed]

9. Paulowicz, I.; Hrkac, V.; Kaps, S.; Cretu, V.; Lupan, O.; Braniste, T.; Duppel, V.; Tiginyanu, I.; Kienle, L.; Adelung, R.; et al. Three-dimensional $\mathrm{SnO}_{2}$ nanowire networks for multifunctional applications: From high-temperature stretchable ceramics to ultraresponsive sensors. Adv. Electron. Mater. 2015, 1, 1500081. [CrossRef]

10. Tiginyanu, I.; Braniste, T.; Smazna, D.; Deng, M.; Schutt, F.; Schuchardt, A.; Stevens-Kalceff, M.A.; Raevschi, S.; Schurmann, U.; Kienle, L.; et al. Self-organized and self-propelled aero-GaN with dual hydrophilic/hydrophobic behaviour. Nano Energy 2019, 56, 759-769. [CrossRef]

11. Mishra, Y.K.; Kaps, S.; Schuchardt, A.; Paulowicz, I.; Jin, X.; Gedamu, D.; Freitag, S.; Claus, M.; Wille, S.; Kovalev, A.; et al. Fabrication of macroscopically flexible and highly porous 3D semiconductor networks from interpenetrating nanostructures by a simple flame transport approach. Part. Part. Syst. Charact. 2013, 30, 775. [CrossRef]

12. Dragoman, M.; Ciobanu, V.; Shree, S.; Dragoman, D.; Braniste, T.; Raevschi, S.; Dinescu, A.; Sarua, A.; Mishra, Y.K.; Pugno, N.; et al. Sensing up to $40 \mathrm{~atm}$ using pressure-sensitive aero-GaN. Phys. Status Solidi RRL 2019, 13, 1900012. [CrossRef]

13. Dragoman, M.; Braniste, T.; Iordanescu, S.; Aldrigo, M.; Raevschi, S.; Shree, S.; Adelung, R.; Tiginyanu, I. Electromagnetic interference shielding in X-band with aero-GaN. Nanotechnology 2019, 30, 34LT01. [CrossRef] [PubMed]

14. Born, M.; Wolf, E. Principles of Optics, 7th (expanded) ed.; Cambridge University Press: Cambridge, UK, 2003.

15. Zhang, X.; Huang, H.; Zhang, Y.; Liu, D.; Tong, N.; Lin, J.; Chen, L.; Zhang, Z.; Wang, X. Phase transition of two-dimensional $\beta-\mathrm{Ga}_{2} \mathrm{O}_{3}$ nanosheets from ultrathin $\gamma-\mathrm{Ga} 2 \mathrm{O} 3$ nanosheets and their photocatalytic hydrogen evolution activities. ACS Omega 2018, 3, 14469-14476. [CrossRef] [PubMed] 
16. Bourque, J.L.; Biesingerb, M.C.; Baines, K.M. Chemical state determination of molecular gallium compounds using XPS. Dalton Trans. 2016, 45, 7678-7696. [CrossRef] [PubMed]

17. Gibbon, J.T.; Jones, L.; Roberts, J.W.; Althobaiti, M.; Chalker, P.R.; Mitrovic, I.Z.; Dhanak, V.R. Band alignments at $\mathrm{Ga}_{2} \mathrm{O}_{3}$ heterojunction interfaces with $\mathrm{Si}$ and $\mathrm{Ge}$. AIP Advances 2018, 8, 065011. [CrossRef]

18. Huang, L.; Feng, Q.; Han, G.; Li, F.; Li, X.; Fang, L.; Xing, X.; Zhang, J.; Hao, Y. Comparison study of $\beta-\mathrm{Ga}_{2} \mathrm{O}_{3}$ photodetectors grown on sapphire at different oxygen pressures. IEEE Photonics J. 2017, 9, 2731625. [CrossRef]

19. Son, H.; Choi, Y.-J.; Hwang, J.; Jeon, D.-W. Influence of post-annealing on properties of $\alpha-\mathrm{Ga}_{2} \mathrm{O}_{3}$ epilayer grown by halide vapor phase epitaxy. ECS J. Solid State Sci. Technol. 2019, 8, Q3024. [CrossRef]

20. Tak, B.R.; Dewan, S.; Goyal, A.; Pathak, R.; Gupta, V.; Kapoor, A.K.; Nagarajan, S.; Singh, R. Point defects induced work function modulation of $\beta-\mathrm{Ga}_{2} \mathrm{O}_{3}$. Appl. Surf. Sci. 2019, 465, 973-978. [CrossRef]

21. Ritter, J.R.; Huso, J.; Dickens, P.T.; Varley, J.B.; Lynn, K.G.; McCluskey, M.D. Compensation and hydrogen passivation of magnesium acceptors in $\beta-\mathrm{Ga}_{2} \mathrm{O}_{3}$. Appl. Phys. Lett. 2018, 113, 052101. [CrossRef]

22. Braniste, T.; Zhukov, S.; Dragoman, M.; Alyabyeva, L.; Ciobanu, V.; Aldrigo, M.; Dragoman, D.; Iordanescu, S.; Shree, S.; Raevschi, S.; et al. Terahertz shielding properties of aero-GaN. Semicond. Sci. Technol. 2019, 34, 12LT02. [CrossRef]

23. Kozlov, G.V.; Volkov, A.A. Millimeter and Submillimeter Wave Spectroscopy of Solids; Gruner, G., Ed.; Springer-Verlag: Berlin, Germany, 1997; p. 51.

(C) 2020 by the authors. Licensee MDPI, Basel, Switzerland. This article is an open access article distributed under the terms and conditions of the Creative Commons Attribution (CC BY) license (http://creativecommons.org/licenses/by/4.0/). 